\title{
Controlled Release of Doxorubicin Loaded within Magnetic Thermo- responsive Nanocarriers under Magnetic and Thermal Actuation in a Microfluidic Channel
}

\author{
Manuel Pernia Leal, ${ }^{\dagger}$ Andrea Torti, ${ }^{\ddagger}$ Andreas Riedinger, ${ }^{\dagger}$ Rocco La Fleur, $^{\ddagger}$ Daniela Petti, ${ }^{\ddagger}$ Roberto Cingolani, ${ }^{\dagger}$ \\ Riccardo Bertacco, ${ }^{\ddagger}$ and Teresa Pellegrino ${ }^{\dagger, \S, *}$ \\ ${ }^{\dagger}$ Istituto Italiano di Tecnologia, Via Morego 30, 16163 Genova, Italy, ' L-NESS and CNISM, Dipartimento di Fisica, Politecnico di Milano-Polo di Como, Via Anzani 42, \\ 22100 Como, Italy, and ${ }^{5}$ National Nanotechnology Laboratory of CNR-NANO, Via per Arnesano km 5, 73100 Lecce, Italy
}

\begin{abstract}
We report a procedure to grow thermo-responsive polymer shells at the surface of magnetic nanocarriers made of multiple iron oxide superparamagnetic nanoparticles embedded in poly(maleic anhydride-alt-1-ocatadecene) polymer nanobeads. Depending on the comonomers and on their relative composition, tunable phase transition temperatures in the range between 26 and $47^{\circ} \mathrm{C}$ under physiological conditions could be achieved. Using a suitable microfluidic platform combining magnetic nanostructures and channels mimicking capillaries of the circulatory system, we

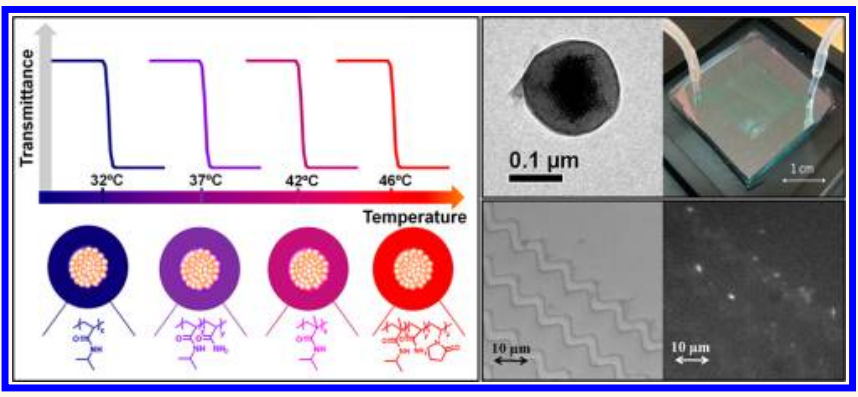
demonstrate that thermo-responsive nanobeads are suitable for localized drug delivery with combined thermal and magnetic activation. Below the critical temperature nanobeads are stable in suspension, retain their cargo, and cannot be easily trapped by magnetic fields. Increasing the temperature above the critical temperature causes the aggregation of nanobeads, forming clusters with a magnetic moment high enough to permit their capture by suitable magnetic gradients in close proximity to the targeted zone. At the same time the polymer swelling activates drug release, with characteristic times on the order of one hour for flow rates of the same order as those of blood in capillaries.
\end{abstract}

KEYWORDS: thermo-responsive $\cdot$ magnetic nanoparticles $\cdot$ drug delivery $\cdot$ microfluids $\cdot$ biomedical applications

$\mathrm{n}$ the field of drug delivery, many efforts
have been undertaken to develop nano-
carriers that can mask the drug mol-
ecules and selectively release the drug
content only at the tumor site. ${ }^{1}$ Although
drug encapsulation has reduced the toxicity
of many drugs, the main problem is still repre-
sented by the actual accumulation of car-
riers at the tumor mass. The presence of
blood flow, together with the dilution of the
carrier in the bloodstream, dramatically re-
duces their accumulation at the tumor dur-
ing the circulation time. Besides passive accu-
mulation mechanisms, such as that based
on the enhanced permeation through tumor
vascular vessels, active retention mechanisms
that rely on the specific targeting of tumor tissues via recognition units present at the nanocarrier surface have been exploited so far. However there is a strong effort in developing alternative ways to remotely enhance drug accumulation. ${ }^{2,3}$ Here we demonstrate a novel method exploiting the local effects of a thermal stimulus on magnetic nanobeads coated with a thermo-responsive polymer to promote particle aggregation and thus preconcentrate the drug carriers before drug release.

Several works have shown how thermoresponsive polymers are able to undergo volume changes at tunable transition temperatures (also called lower critical solution temperature, LCST), thus passing from being colloidally stable and disperse to
* Address correspondence to teresa.pellegrino@unisalento.it.

Received for review June 26, 2012 and accepted November 1, 2012.

Published online

10.1021/nn3028425

C XXXX American Chemical Society 
collapsed and sticky configurations, which generate polymer aggregates. ${ }^{4-7}$ This aggregation process is fully reversible: by lowering the temperature below the critical value, the reverse phase transition occurs, leading to disaggregation of the thermo-responsive polymers. ${ }^{8}$ This process might be exploited to locally accumulate nanocarriers in proximity of a tumor site. Indeed, due to the sizable increase of the hydrodynamic volume and magnetic moment associated with the clusters, efficient focusing via magnetic field gradients can be easily achieved. In addition, the shrinking of the thermo-polymer can be also exploited for triggering the (temperature-mediated) release of drug molecules previously loaded within the polymer networks.

The association of soft materials with iron oxide nanoparticles (IONPs) to build up multifunctional nanocarriers represents an ideal solution allowing for the external activation of a thermo-responsive polymer. So far, IONPs were shown to be useful heat foci in hyperthermia tumor treatment acting under alternating magnetic fields of appropriate frequencies and amplitudes. ${ }^{9-12}$ Such nanocrystals present an additional advantage as drug delivery tools: due to their superparamagnetic behavior at body temperature, they do not present any remanent magnetization, and consequently the magnetic nanocarriers do not experience aggregation unless an external magnetic field is applied locally, in the region to be treated. ${ }^{13-16}$ Entrapping several IONPs into the same polymer nanoparticle has the further benefit of facilitating its manipulation, requiring lower magnetic fields than in the case of single polymer coated IONPs. ${ }^{17}$

To date, different magnetic thermo-sensitive nanosystems have been developed based on liposomes, ${ }^{18}$ hydrogels, ${ }^{19}$ micelles, ${ }^{20}$ nanobeads, ${ }^{21}$ particles, ${ }^{22}$ etc., and different limitations have been already underlined. ${ }^{23}$ For applications in drug delivery, indeed the triggered temperature of the polymer should be higher than body temperature $\left(37^{\circ} \mathrm{C}\right)$, in order to avoid leakage of the drug from the nanocarriers before reaching the target. Remarkably, the swelling of the polymer depends on parameters such as the salt concentrations and the $\mathrm{pH}$ of the medium. Therefore the choice of the medium in which the polymer is analyzed is crucial: for instance, the swelling of the same polymer nanoparticle is significantly different in pure Milli-Q water and in physiological solution. ${ }^{24,25}$

However, the behavior of such multifunctional magnetic thermo-responsive nanocarriers in fluidic channels that simulate the bloodstream condition is not yet fully elucidated. ${ }^{26,27}$ The investigation of the motion of nanocarriers and the efficiency of their capture by controllable magnetic poles embedded in microfluidic platforms is the first step in view of in vivo application of nanocarriers as drug delivery tools through capillaries of the circulatory systems surrounding tumor cells, where a local magnetic gradient can be generated for concentrating the carriers. Bertacco and coworkers have recently developed a technological platform for the controlled trapping, transport, and release of magnetic particles in suspension over magnetic stripes (conduits) integrated in microfluidic systems. ${ }^{16}$ In this approach magnetic domain walls (DWs) can be generated at specific pinning sites of the conduits within a microfluidic channel via external magnetic field pulses. Each DW generates a highly localized magnetic field gradient that is capable of trapping micro- and nanosized magnetic carriers. ${ }^{28,29}$ Herein, the same platform is used to investigate the clustering induced by the temperature and the subsequent magnetic trapping promoted by the aggregation.

In the present study, we report a procedure to fabricate magnetic thermo-responsive nanocarriers based on multiple IONPs embedded in a polymeric double shell, which includes an internal one made of poly(maleic anhydride-alt-1-ocatadecene) and an outer shell composed of thermo-responsive polymers. Depending on the choice of comonomers and on their relative molar ratio, tunable phase transition temperatures in the range between 26 and $47{ }^{\circ} \mathrm{C}$ have been achieved in physiological conditions. Furthermore, we demonstrate the possibility to magnetically manipulate our magnetic thermo-responsive nanobeads in the microfluidic chip under thermal actuation. Doxorubicin (DOXO) as a model drug was loaded by physical absorption within the thermo-responsive shell, and the release of DOXO from the magnetic nanosystems was performed in a microfluidic channel on a chip with zigzag-shaped permalloy $\left(\mathrm{Ni}_{80} \mathrm{Fe}_{20}\right)$ microstripes. At a fixed flow rate inside the channel the DOXO-loaded magnetic thermo-responsive nanocarriers can be trapped by DWs only above the LCST (at temperature values slightly above body temperature). Increasing the temperature above the LCST promotes both aggregation and drug release, the latter being strongly dependent on the flow rate inside the channel.

\section{RESULTS AND DISCUSSION}

Synthesis of Thermo-responsive Polymers. As recently reported by some of us, polymeric magnetic nanobeads having a diameter below $100 \mathrm{~nm}$ can be fabricated by the solvent destabilization of alkyl polymaleic anhydride polymer molecules and IONPs. ${ }^{30,31}$ In a further development, by a two-step approach, we have grown a poly- $N$-isopropylacrylamide (pNIPAM) polymer shell at the bead surface by first priming the nanobead surface with amino ethyl methacrylate (AEM) molecules, thus introducing $\mathrm{C}-\mathrm{C}$ double bonds on which the polymerization can propagate. Later, we copolymerized $\mathrm{N}$-isopropylacrylamide (NIPAM) to the nanobead surface resulting in pNIPAM-magnetic nanobeads exhibiting a LCST of $32{ }^{\circ} \mathrm{C}$ in water, typical of this polymer microgel. ${ }^{21}$ This phase transition temperature 


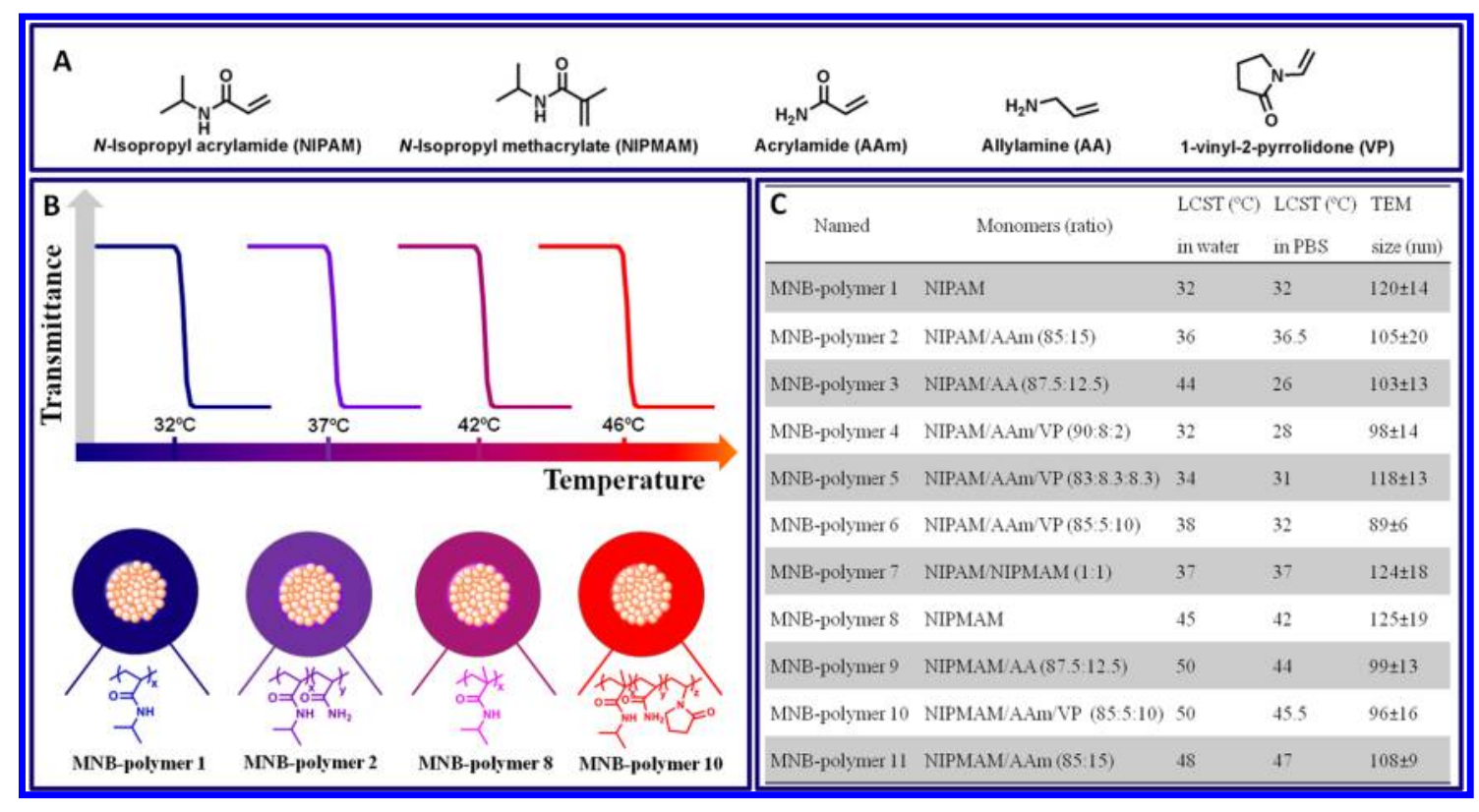

Figure 1. (A) Monomers used in the polymer synthesis at the magnetic nanobead surface. (B) Schematic cartoons of the structure and phase transition temperatures of some of the MNB-polymers. (C) TEM diameter sizes of MNB-polymers and LCSTs of synthesized MNB-polymers measured in water and in $50 \mathrm{mM}$ PBS, $154 \mathrm{mM} \mathrm{NaCl}$.

makes the pNIPAM-magnetic nanobeads unsuitable for clinical applications, as it is lower than body temperature.

The copolymerization of NIPAM with different hydrophilic monomers can shift the phase transition temperatures of the resulting polymer from $32{ }^{\circ} \mathrm{C}$ to higher temperature values. ${ }^{5}$ For instance, the copolymerization of NIPAM with monomers such as 1-vinyl2-pyrrolidone (VP), acrylamide (AAm), allylamine (AA), $\mathrm{N}$-vinylacetamide (NVA), methacrylamide (MAAm), $\mathrm{N}$-isopropylmethylacrylamide (NIPMAM), and N-methyl$\mathrm{N}$-vinylacetamide (MVA) has provided polymer microgels having LCSTs up to $46^{\circ} \mathrm{C}^{32}$ Furthermore, microgel polymers based on ternary monomer composition such as NIPAM, acrylic acid (AAC), and ethyl methacrylate (EMA), ${ }^{24}$ or NIPAM, AAm, and VP ${ }^{25}$ were shown to change the hydrophobic/hydrophilic balance of bare pNIPAM and raise the phase transition temperatures from $25^{\circ} \mathrm{C}$ to $70{ }^{\circ} \mathrm{C}$ under physiological conditions.

Inspired by these works and with the intent to increase the LCST of the resulting magnetic beads above $37^{\circ} \mathrm{C}$, herein we have developed a protocol to copolymerize at the surface of magnetic beads different monomers (see Figure 1A). Due to the different nature of the monomers chosen and in order to grow a stable polymer shell composed of more than one monomer type, the synthesis conditions had to be refined with respect to our previous works. The amount of AEM molecules added per bead was found to be critical in order to avoid the main problem of nanobead precipitation during the synthesis. An overfunctionalization of methacrylate groups, indeed, led to a precipitation of the beads during the first step of the reaction. This can be likely ascribed to the increase in the hydrophobicity of the nanobead surface. On the other hand, a poor methacrylate functionalization resulted in stable nanobeads in both acetonitrile and aqueous solvents during the first reaction step, but later during the polymerization of the comonomers, the nanobeads precipitated out of the solution. The reasons for this aggregation could possibly be related to the low density of grafting methacrylate groups at the surface of the nanobeads, which promotes interparticle cross-linking rather than polymer growth at each nanobead. Moreover, in water at $\mathrm{pH} 6.8$ a large number of carboxylate groups at the nanobead surface are deprotonated as well as some comonomers (this is likely the case for acrylic acid), thus leading to repulsive interactions among them. These forces might impair the starting of the polymerization of the monomers at the bead surface with a random polymerization of monomers occurring in solution and consequent aggregation of nanobeads entrapped within the polymer.

We found that the range in which the amount of AEM could be varied depends on the size and the concentration of nanobeads. For instance, AEM in a range of $0.14-0.22 \mathrm{mmol}$ was added to $1 \mathrm{~mL}$ of a solution of magnetic nanobeads (bead TEM diameter of $66 \pm 9 \mathrm{~nm}$, which corresponds to an iron concentration of $529 \mathrm{mg} / \mathrm{L}$ and $5.4 \times 10^{-6} \mathrm{~mol}$ of beads), thus leading to stable beads during the entire synthetic pathway and allowing, in the second reaction step, the copolymerization of different monomers (see Figure 1).

In accordance with a previous work in which the copolymerization of acrylamide with NIPAM at different molar ratios can provide microgels with LCSTs in the range $36-42{ }^{\circ} \mathrm{C}^{32,33}$ we used a NIPAM/AAm molar ratio of $85: 15$, which should provide a thermo-responsive 


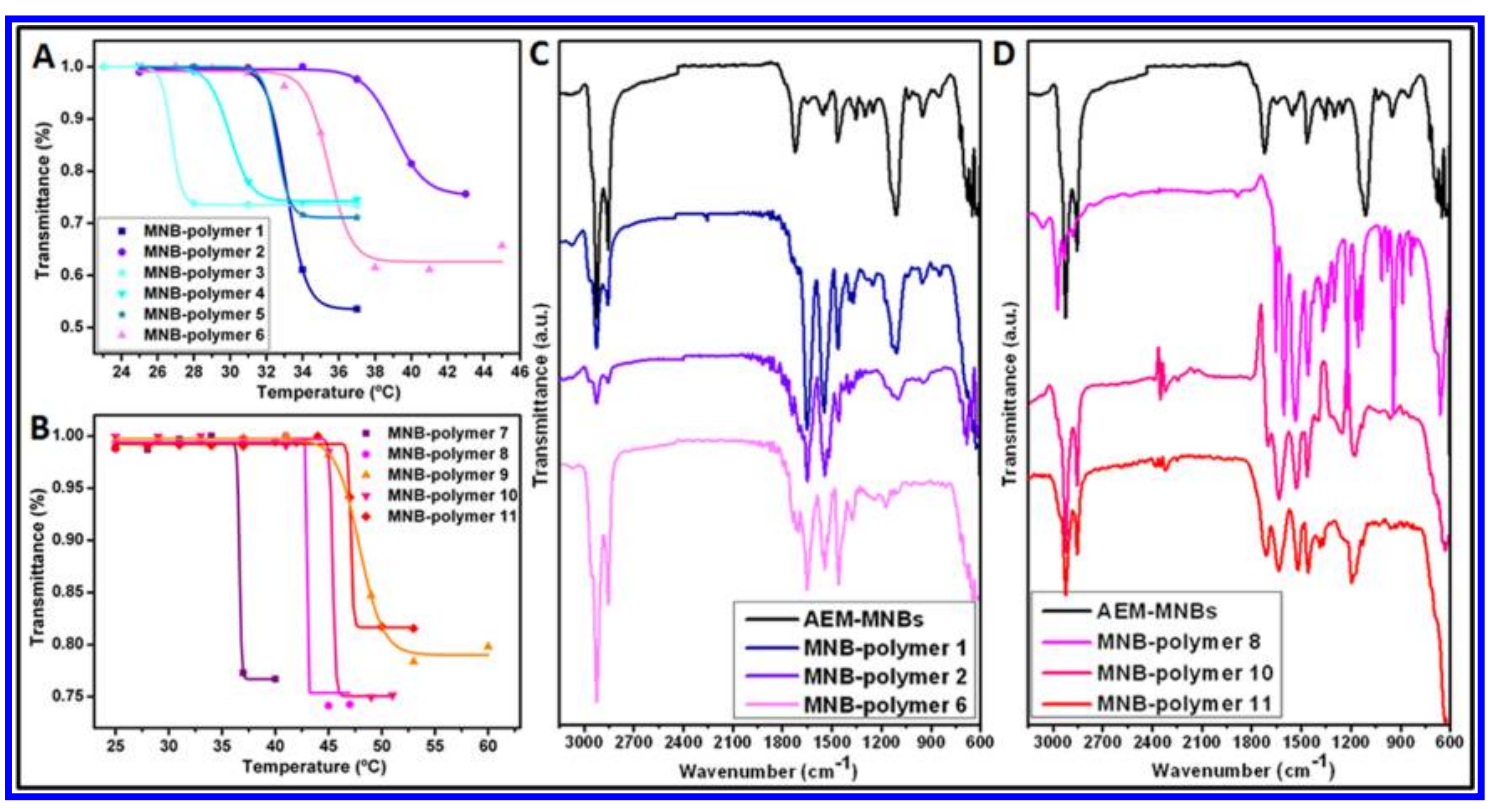

Figure 2. (A) Cloud point measurements of pNIPAM-derived polymers and (B) pNIPMAM-derived polymer. (C) FTIR spectra of pNIPAM- and (D) pNIPMAM-derived polymers.

shell at the bead surface with an LCST of $42{ }^{\circ} \mathrm{C}$. In this case, the obtained NIPAM/AAm-MNBs (hereafter named MNB-polymer 2) were stable in solution, and an increase of the TEM bead diameter from $66 \pm 9 \mathrm{~nm}$ to $105 \pm 20 \mathrm{~nm}$ provided an indication of the polymer growth at each nanobead surface. The LCST measured by the curve of the transmittance $v$ s temperature (Figure 2A and B) for the MNB-polymer 2, however, showed that the decay of the phase transition started at $36.5^{\circ} \mathrm{C}$ in $50 \mathrm{mM} \mathrm{PBS}$ buffer solution (pH 7.2, $154 \mathrm{mM}$ $\mathrm{NaCl}$ ), still lower than the body temperature and lower than the temperature expected for this NIPAM/AAm molar ratio. This temperature difference might be due to the effective composition of the NIPAM/AAm at the nanobead surface, which might diverge from the initial amount of NIPAM/AAm added in solution for the copolymerization. As reported in the literature, ${ }^{34}$ the mixture of NIPAM/allylamine could result in higher LCSTs, and thus a molar ratio of 87.5:12.5 was chosen for the copolymerization onto the nanobead surface. The resulting nanobeads (MNB-polymer 3 ) were stable and monodisperse, and as expected, due to the polymer growth, an increase in the TEM diameter from $66 \pm$ $9 \mathrm{~nm}$ to $103 \pm 13 \mathrm{~nm}$ was recorded, but an unexpected decay of the phase transition temperature at $26^{\circ} \mathrm{C}$ was obtained. This temperature difference was likely due to the fact that the LCST depends on physiological conditions, and the LCST of $42^{\circ} \mathrm{C}$ reported in the literature was measured on the polymer microgel in water (indeed when we measured the LCST in water, we found a phase transition temperature of $44^{\circ} \mathrm{C}$ even on the MNB-polymer 3; see Figure 1C). Ternary monomer mixtures based on NIPAM/AAm and VP were also reported as thermo-responsive polymer compositions which raise the LCSTs to values higher than $37{ }^{\circ} \mathrm{C}$ depending on the molar ratio of monomers. ${ }^{25}$ At a ratio of 90:8:2 of respectively NIPAM/AAm/VP the resulting nanobeads (MNB-polymer 4) were stable and monodisperse, with a TEM diameter of $98 \pm 14 \mathrm{~nm}$, but the LCST was just slightly shifted to $28^{\circ} \mathrm{C}$. At a molar ratio of 83.3:8.3:8.3, which corresponded to a higher amount of VP in the starting composition of the hydrophilic monomers, stable nanobeads (MNB-polymer 5) with a larger TEM diameter peaking at $118 \pm 13 \mathrm{~nm}$ and with a phase transition temperature of $31^{\circ} \mathrm{C}$ were obtained. Finally, at a molar ratio of 85:5:10 of respectively NIPAM/AAm/VP, MNB-polymer 6 displayed an LCST of $32{ }^{\circ} \mathrm{C}$ in PBS buffer. A further increase in the composition of the VP monomers did not result in a stable nanobead solution. It is also worth mentioning that when another ternary monomer composition consisting of NIPAM, AAC, and EMA, already proposed in the literature for producing thermo-responsive microgels with an LCST higher than body temperature, was employed to coat the nanobeads, the nanobeads collapsed during the polymerization (as explained before, repulsive interactions between deprotonated AAC and methacrylated-functionalized MNBs might be the cause of this precipitation). We should underline that in general we have also observed a drop in the LCST measured on the thermo-responsive coated MNBs with respect to the LCST of microgel polymer reported in the literature when similar comonomer compositions were used. ${ }^{25,32-35}$ These differences could be explained by the effect of the $\mathrm{pH}$ and salts on the LCST. These results showed that the LCST depends on (i) the chemical composition (referring to the different monomer ratios added at the nanobeads), (ii) the $\mathrm{pH}$ of the solution, which plays an important role in the degree of protonation of the monomers, and (iii) the salt 
concentration, which can also play a role in the solvatation of charged groups as reported before. ${ }^{24,25}$ For instance, the presence of comonomer allylamine in the MNB-polymer 3 was affecting the LCST by the presence of salts and by the $\mathrm{pH}$ of the media; it dropped from $44{ }^{\circ} \mathrm{C}$ to $26{ }^{\circ} \mathrm{C}$ when water was exchanged with PBS (Figure 1C).

In order to raise the LCST, another monomer named $\mathrm{N}$-isopropyl methacrylate (NIPMAM), ${ }^{36}$ rather than NIPAM, was chosen as the main monomer. As reported in the literature, despite that this polymer is slightly more hydrophobic than NIPAM, so a lower LCST should be expected, the higher phase transition temperature recorded could be due to the steric hindrance induced by the methyl group, thus preventing joining the hydrophobic groups together in the most favorable way as reported in the literarure. ${ }^{36}$ The polymerization of NIPMAM with other comonomers such as NIPAM, $A A, A A m-V P$, and $A A m$ led to the polymerization of the thermo-responsive shell at the bead surface with phase transition temperatures in physiological conditions tunable from 37 to $47{ }^{\circ} \mathrm{C}$ (Figure 1) (TEM diameters of the nanobeads are available in Figure $1 \mathrm{C}$ and Figures $1 \mathrm{~S}$ and $2 \mathrm{~S}$ of the Supporting Information, $\mathrm{SI}$ ).

In general, the LCSTs from NIPMAM-based magnetic nanobeads in PBS show temperatures above $37{ }^{\circ} \mathrm{C}$, with sharp phase transitions with respect to the LCSTs in water (Figure 2B and Figure 3S). The best nanobead-thermo-responsive polymer composition for our proposal is the NIPMAM-coated nanobeads (MNB-polymer 8), which possess a characteristic LCST from 41 to $42{ }^{\circ} \mathrm{C}$ (magenta curve in Figure 2B) in physiological conditions. Indeed, the characterization and capacity to load and release doxorubicin as a chemotherapeutic agent in MNB-polymer 8 will be discussed in detail in the following parts.

Characterization of Thermo-responsive Polymers. The compositional characterization of the polymer shell grown around the magnetic nanobeads was performed by FT-IR spectroscopy. The spectrum of the amino ethyl methacrylate nanobeads (AEM-MNBs) (black curve in Figure $2 \mathrm{C}$ and D) showed a broad signal at $1720 \mathrm{~cm}^{-1}$ coming from the carboxylate (stretching $\mathrm{C}=\mathrm{O}$ ) and several peaks at $1650 \mathrm{~cm}^{-1}$ (amide I) and at $1550 \mathrm{~cm}^{-1}$ (amide II) due to the stretching and bending of a secondary amide, respectively $(\mathrm{C}=\mathrm{O} / \mathrm{N}-\mathrm{H})$, and at $1468 \mathrm{~cm}^{-1}$ likely due to the stretching of the $\mathrm{C}-\mathrm{N}$ bond. After polymerization some signals are much stronger because of the increase of the amide bonds in the nanobead shell and some of the signals were slightly shifted. For instance, the signals around 1650 and at $1550 \mathrm{~cm}^{-1}$ were shifted at 1635 or $1645 \mathrm{~cm}^{-1}$, and $1530 \mathrm{~cm}^{-1}$, respectively, likely due to the internal hydrogen bonds between the carbonyl and the amide groups (Figure 2C, D and Figure 4S, SI). For the same reason the signal at $1465 \mathrm{~cm}^{-1}$ increased as well, which corresponds to the $\mathrm{C}-\mathrm{N}$ stretching. In addition, two signals at 1390 and $1330 \mathrm{~cm}^{-1}$ deriving from the isopropyl group were observed in both cases where NIPAM or NIPMAM was the main monomer in the polymer composition. Furthermore, the higher the initial molar ratio of NIPAM or NIPMAM with respect to the other monomers, the more intense the signal at $1530 \mathrm{~cm}^{-1}$, indicating increasing amounts of NIPAM or NIPMAM at the nanobead surface. It is worth mentioning that while FTIR spectra can provide qualitative indications about the different polymer composition at the bead surface, with this technique, it was not possible to quantify the copolymer composition, and on the other hand, NMR spectroscopy, which could provide this information, cannot be performed on the magnetic nanobeads due to the superparamagnetic core.

As a further confirmation of the presence of polymer shell at the bead surface at different compositions, we have monitored the hydrodynamic diameters of the thermo-responsive polymers by dynamic light scattering (DLS) at temperatures well below and well above the LCST of the respectively MNB-polymer (Figure 3A). In physiological conditions, all the MNBpolymers showed a negative volume response when the temperature reached their phase transition; for instance the volume of MNB-polymer 1 was reduced about $40 \%$ when the temperature was brought from $25^{\circ} \mathrm{C}$ to $32{ }^{\circ} \mathrm{C}$. Generally, we observed that the shrinking capacity of the NIPAM polymer-nanobeads is much higher than those of the NIPMAM polymer, and one possible explanation for this difference could be the presence of an extra methyl group in the structure of the NIPMAM, which induces more hindrance to the polymer system, thus decreasing the bound water volume between the polymer chains.

Furthermore the TEM characterization, besides providing a diameter increase after the polymer shell growth, also proved that the thermo-responsive polymer nanobeads were less sticky and were also well separated on the TEM grids with respect to the initial AEM-MNBs (Figure 3C and D and Figure $1 \mathrm{~S}$ in the $\mathrm{SI}$ ).

Finally, it is also worth mentioning that when the temperature of a MNB-polymer solution (at a concentration of $50 \mathrm{mg} / \mathrm{L}$ in $\mathrm{Fe}$ ) was brought from below to above the LCST value as a consequence of the polymer shrinking, a macroscopic precipitation of the nanobeads visible by the bare eye was observed (as an example see Figure 3B). This state is completely reversible, and when the temperature was brought back below the phase transition temperature, the nanobeads were fully redissolved in the solution. The reversibility of the process was also observed by comparing the hydrodynamic diameter of the MNBpolymer 8 at temperatures below and above the LCST. After a few cycles, the MNB-polymer loses complete flexibility, thus preventing a full recovery of its initial hydrodynamic size (Figure 5S, S.I.).

Loading and Release of Doxorubicin. To investigate the potential of our system for controlled drug loading and 


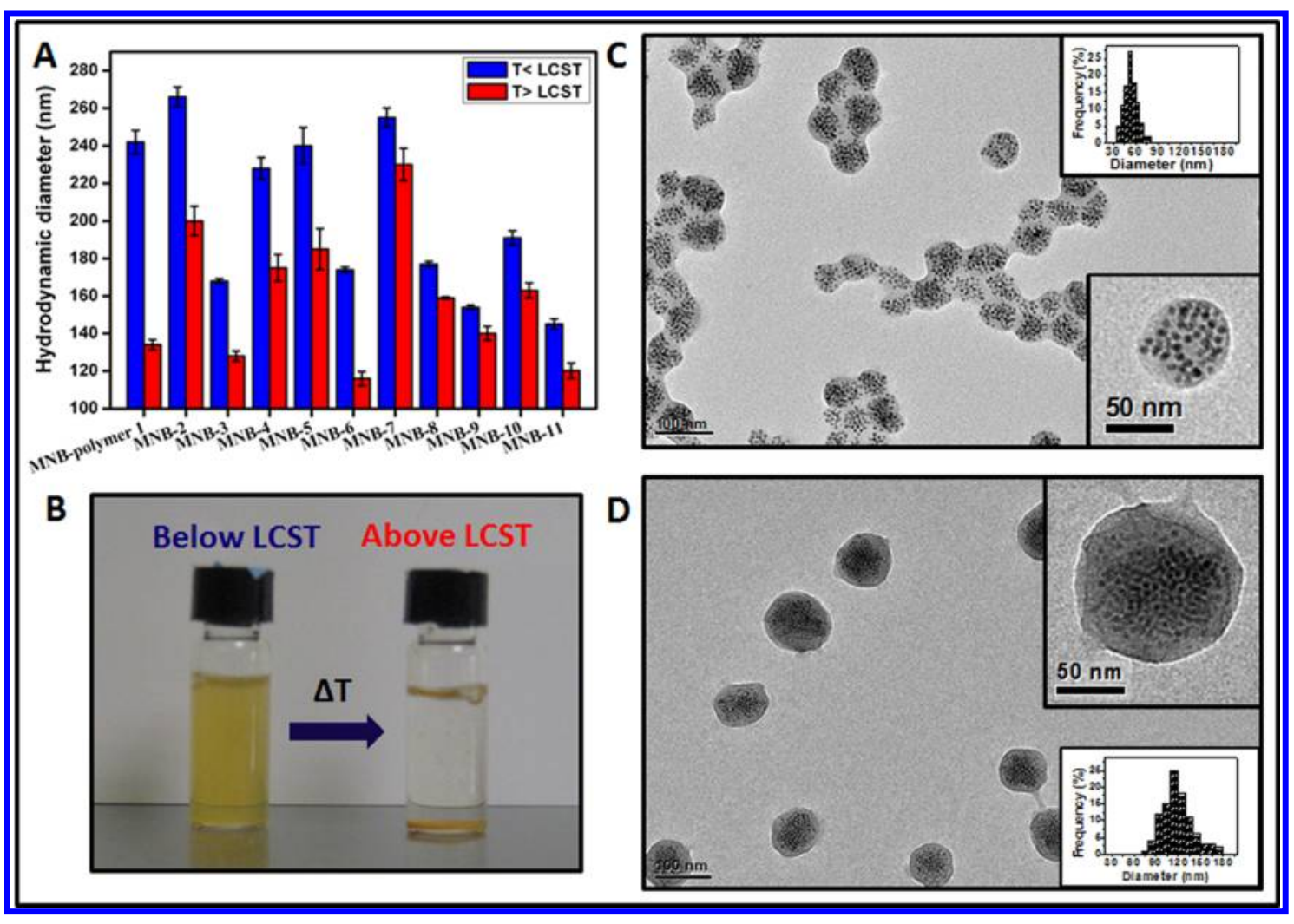

Figure 3. (A) Hydrodynamic radii below (blue bar) and above (red bar) the phase transition temperature of the different synthesized thermo-responsive magnetic nanobeads (see also Table 1S in the SI). (B) Comparison of MNB-polymers below and above their LCSTs. (C) Representative TEM images of AEM-MNBs, the first step of reaction, and (D) MNB-polymer 8 after polymer 8 growth at the nanobead surface. The insets represent the corresponding histograms of the TEM size measured on at least 100 nanobeads. Note: The agglomeration observed in part $\mathrm{C}$ is just an artifact of the drying effect of the polymer beads on the TEM grid, as already verified in ref 17 (see also Figure S13 in the $\mathrm{SI}$ ).

release, the MNB-polymers 8,9 , and 10 exhibiting an LCST of 42,44 , and $45.5^{\circ} \mathrm{C}$, respectively, were chosen, as they remain stable below $40^{\circ} \mathrm{C}$ and thus also at body temperature. In order to simulate the temperatureactivated trapping and controlled release of the drug molecules at a specific point inside the blood vessels, a microfluidic channel made of PDMS was fabricated on top of a $\mathrm{SiO}_{2} / \mathrm{Si}$ chip with zigzag-shaped magnetic structures suitable for the stabilization of a magnetic domain wall in each corner via application of an external magnetic field $(50 \mathrm{mT})$ perpendicular to the main axis of the stripes (Figure $4 \mathrm{~A}$ ).$^{28}$ Due to the strong intensity and gradient of the magnetic field generated by the DWs, they act as efficient trapping points for magnetic particles inside the microfluidic channel, which can be also easily erased by applying a magnetic field with the same intensity along the main axis of the stripes. Doxorubicin, a drug now applied in chemotherapy, was chosen as drug reference and loaded into the MNB-polymers 8, 9, and 10. The DOXO in PBS buffer was mixed with a PBS solution containing the corresponding MNB-polymer, and the mixture was shaken overnight at room temperature. The DOXO indeed can be physically absorbed within the nanogel polymer networks. ${ }^{37}$ After that, the DOXO-loaded MNB-polymer was centrifuged at $4200 \mathrm{rpm}$ for $5 \mathrm{~min}$, and additionally the vial was placed close to a permanent magnet $(0.3 \mathrm{~T})$ in order to quantitatively separate the magnetic nanobeads from the solution containing free DOXO. To quantify the amount of DOXO loaded with the nanobeads, the absorption spectra of the collected supernatants after the magnet purification were recorded (Figures $6 \mathrm{~S}$ and $7 \mathrm{~S}, \mathrm{SI}$ ). The DOXO loading efficiency for the MNB-polymer 8 was calculated to be $90.6 \%$, resulting in a final DOXO concentration of $68 \mu \mathrm{g} / \mathrm{mL}$ per MNB-polymer 8 at a concentration of $[\mathrm{Fe}]=50 \mathrm{mg} / \mathrm{L}$; that for the polymer MNB-polymer 9 was $41.8 \%$ $(31.4 \mu \mathrm{g} / \mathrm{mL}$ per $[\mathrm{Fe}]=50 \mathrm{mg} / \mathrm{L})$ and $67.2 \%$ for MNBpolymer $10(50.4 \mu \mathrm{g} / \mathrm{mL}$ per [Fe] $=50 \mathrm{mg} / \mathrm{L})$.

For investigating the controlled release of DOXO, the DOXO-loaded MNB-polymer 8 suspended in PBS was injected via a syringe pump at a constant flow rate of $1 \mu \mathrm{L} / \mathrm{min}$ in a microfluidic channel set at $25^{\circ} \mathrm{C}$ for the control experiment and at $45^{\circ} \mathrm{C}$ for the release experiment. Then, magnetic DWs were created at each corner of the magnetic microstripes at the bottom of the microfluidic channel in PDMS, by applying an in-plane magnetic field pulse of $50 \mathrm{mT}$ perpendicular to the long axis of the structures. At $25^{\circ} \mathrm{C}$ the magnetic stray field generated by the DWs was not able to trap single nanobeads, thus indicating that below the LCST the magnetic moment of the MNBs is too small to ensure 


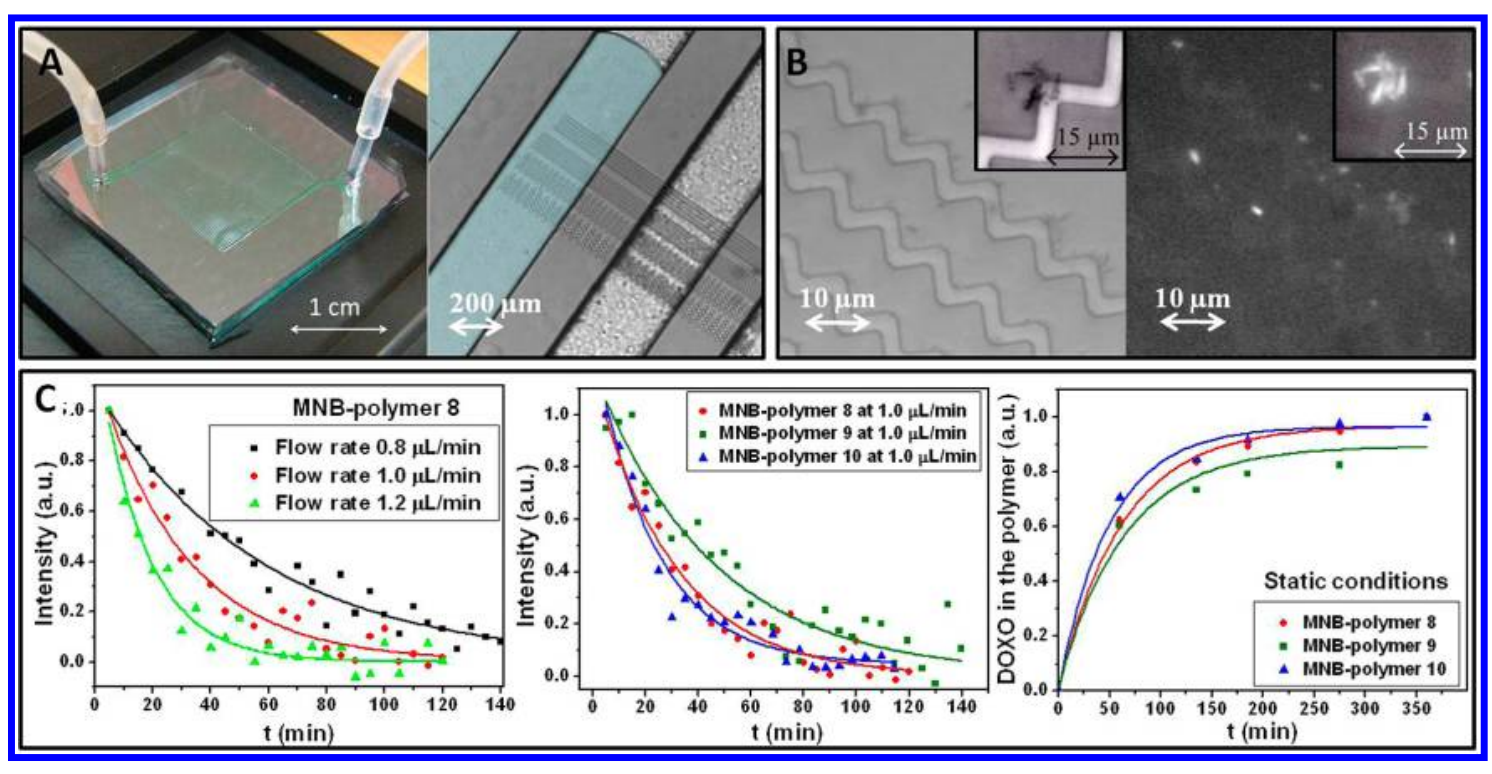

Figure 4. (A) Image of a typical chip used in the experiment (left panel) and of the microfluidic channels with zigzag-shaped permalloy $\left(\mathrm{Ni}_{80} \mathrm{Fe}_{20}\right)$ microstripes (right panel). The portion of the channel filled with liquid has been colored in light blue in the left portion of panel, while the light gray regions correspond to the empty portion of the serpentine. (B) Bright field (BF) image of the aggregates made of MNB-polymer 8 at $45^{\circ} \mathrm{C}$ trapped by the DWs at several corners of one of the zigzag-shaped magnetic conduits (left panel) and corresponding FITC filtered image showing the fluorescence of the aggregates, due to the doxorubicin molecules encapsulated within the magnetic nanobeads (right panel). The insets show the zoom-in of a single trapped polymeric aggregate in the BF and FITC filtered image. (C) Normalized fluorescence intensity vs time curves of the polymeric complex MNB-polymer 8 trapped on a DW site at different flow rates (left panel); normalized fluorescence intensity vs time curves of polymeric complexes MNB-polymer 8,9 , and 10 trapped on a DW site at $1.0 \mu \mathrm{L} / \mathrm{min}$ flow rate (central panel); cumulative release profile of DOXO from MNBs coated with polymers 8,9 , and 10 in the absence of any flow rate (right panel).

capturing. MNBs are stable in suspension and the magnetic force is not enough to overcome surface forces even if the flow rate is lowered to $0.5 \mu \mathrm{L} / \mathrm{min}$. Below the LCST, single DOXO-loaded MNB-polymer 8 particles flow essentially unperturbed in the channels, and their motion can be easily recorded, especially looking at the fluorescent signal, as shown in Figure 8S (taken from Video 1S in the SI). Subsequently, the chip substrate was set at $45^{\circ} \mathrm{C}$, above the LCST of the MNBpolymer 8 . At this temperature, the polymer 8 becomes hydrophobic and forms agglomerates in solution (see Figure 3B), bringing together a large number of magnetic nanobeads (Figure 4B). These aggregates were captured by the array of magnetic trapping points localized at each corner of the zigzags (see Video $2 \mathrm{~S}$ in the $\mathrm{SI}$ ), since the magnetic interaction between the DWs and the aggregates is strengthened due to the larger magnetic moment of the latter one. This is clearly visible in Figure 4B, where we show a brightfield image of the structures with some captured beads (dark spots) and a fluorescence image acquired simultaneously where the fluorescent beads are evident. The second relevant effect of the increase in temperature above the phase transition value of the MNBpolymer 8 is the shrinkage of the polymer, which induces cargo release. In order to study the time evolution of the DOXO release, we have recorded the fluorescence decay of individual DOXO-loaded MNBpolymer 8 starting from the moment of trapping on a DW, at different flow rates $(0.8,1.0$, and $1.2 \mu \mathrm{L} / \mathrm{min}$,
Figure 4C (left panel) and Figure 9S in the SI). It was noticed that an increase in the flow rate led to a strong decrease of the fluorescence intensity time decay, while the MNBs were not detached from the position where they were trapped (Figure $10 \mathrm{~S}$ of the $\mathrm{SI}$ ). At $0.8 \mu \mathrm{L} / \mathrm{min}$ the decay time was $57 \pm 8 \mathrm{~min}$. Increasing the flow rate to $1.0 \mu \mathrm{L} / \mathrm{min}$ led to a decay time of $31 \pm$ $4 \mathrm{~min}$, while an additional increase up to $1.2 \mu \mathrm{L} / \mathrm{min}$ gave a decay time of only $17 \pm 2 \mathrm{~min}$. Different DOXOloaded MNB-polymers were tested in the microfluidic channels for comparing whether their release capacity depends on the polymer composition. The MNB-polymer 9 based on the copolymer NIPMAM and allylamine (87.5:12.5) and the MNB-polymer 10 based on terpolymers NIPMAM, acrylamide, and vinylpyrrolidone (85:5:10) were injected at constant flow rate of $1 \mu \mathrm{L} / \mathrm{min}$ in the microfluidic channels set at 46 and $47^{\circ} \mathrm{C}$, i.e., above the LCST of the MNB-polymers 9 and 10 , respectively (central panel Figure $4 C$, Figure $9 S$ in the $\mathrm{SI}$ ). The DOXO release profiles of these MNB-polymer formulations were quite similar to the MNB-polymer 8 , with the decay times in the same range $(38 \pm 6 \mathrm{~min}$ for the MNB-polymer 9 and $28 \pm 4$ min for the MNB-polymer 10), while the activation LCST temperature was different. These data suggest that a different composition in the polymer shell at the surface of the magnetic nanobeads does not significantly affect the rate of the release.

The reversibility of the thermally induced aggregation process has been investigated by observing the 
progressive disaggregation of trapped aggregates upon reduction of the temperature below the LCST (see Video $3 \mathrm{~S}$ in the SI.) A clear tendency to disaggregation is visible, leading to the spreading of the fluorescence signal over a large area of the chip, elongated along the direction of the liquid flow. It is worth mentioning that in order to avoid the photobleaching, the light shutter was opened for only $10 \mathrm{~s}$ and each data point was collected at 5 min intervals. To exclude any influence of photobleaching, a control experiment has been performed. A solution of DOXO in PBS buffer $(22 \mu \mathrm{g} / \mathrm{mL}$ ) was injected into the microfluidic channel, and then the light intensity decay was monitored every $5 \mathrm{~min}$ for $1 \mathrm{~h}$ at $45^{\circ} \mathrm{C}$ with a constant flow rate of 1 $\mu \mathrm{L} / \mathrm{min}$. Importantly the camera parameters were set at similar values to those used in the release experiments above. In this experiment, any exponential decay of the fluorescence was not observed (Figure 11S, SI), thus confirming that the decay of the fluorescence signal from individual aggregates captured by DWs in the microfluidic channels was really due to the release of DOXO molecules and not to the photobleaching of DOXO. To confirm the reliability of these results on individual particles and establish a connection with the asymptotic behavior in static conditions, the DOXO release in a solution of DOXO-loaded nanobeads kept in a vial was measured by UV-vis spectroscopy. A solution of DOXO-loaded MNB-polymers 8, 9, and 10 in PBS buffer was incubated at $47^{\circ} \mathrm{C}$, and after certain time lapses the nanobeads were precipitated out of the solution and captured with a permanent magnet. The supernatants were collected, and the absorption spectra were recorded. After that the MNB-polymers 8, 9, and 10 captured by the magnet were redissolved in the same supernatant, and the incubations were continued. This step was repeated until enough data points were obtained. As expected, the decay times ( $70 \pm 10$, $99 \pm 19$, and $51 \pm 13 \mathrm{~min}$, respectively) are much longer than that measured in the case of the different flow rates imposed in the microchannel (Figure 4C, right panel). This confirms the reliability of results taken within the microfluidic channel and definitely indicates a strong dependence of the drug release on the local flow rate, as shown in Figure $12 \mathrm{~S}$ in the Supporting Information.

\section{CONCLUSIONS}

Herein, we have demonstrated the possibility to tune the phase transition temperature of polymerdecorated magnetic nanobeads, in the range between 26 and $47^{\circ} \mathrm{C}$, by modifying the monomer composition at the nanobead surface. Due to the strong influence on the polymer shrinking behavior of the local temperature, $\mathrm{pH}$, and salt concentration, we identify polymer compositions at the magnetic beads that allow polymer shrinking at LCSTs above body temperature and under physiological conditions. Contemporary aggregation of nanobeads and cargo release is achieved at temperatures slightly above body temperature, thus making these carriers suitable for in vivo localized treatment of diseases such as tumors. The performances of this platform were tested in microfluidic channels fabricated on Si chips and mimicking the capillaries of the circulatory system, ${ }^{38}$ where the nanocarriers could be focused by a localized magnetic field gradient and thermally activated. Nanobead aggregation and capture at the corners of zigzag magnetic conduits was induced by increasing the temperature to $45^{\circ} \mathrm{C}$, i.e., above the LCST. By recording the release profile of an anticancer drug (DOXO) from individual aggregates inside a microfluidic channel under different constant flow rates, we found that realistic release times (on the order of $1 \mathrm{~h}$ ) can be achieved by properly tuning the polymer properties, at a typical flow rate of about $0.8 \mu \mathrm{L} / \mathrm{min}$ in a channel with $65 \mu \mathrm{m}$ height and $270 \mu \mathrm{m}$ width. As these numbers are compatible with the typical dimensions and flow rates in capillaries, our results demonstrate the potential of such magnetic thermo-responsive carriers for future in vivo applications. Provided this first proof-of-concept of thermal aggregation and magnetic entrapment under dynamic conditions, as a further step we aim to promote the magnetic aggregation of these thermoresponsive magnetic nanobeads by irradiation with ac magnetic fields, thus achieving a remote actuation via a hyperthermia effect.

\section{MATERIALS AND METHODS}

Chemicals and General Methods. Reagents were obtained from commercial suppliers and used without further purification. Poly(maleic anhydride-alt-1-octadecene), $M_{\mathrm{n}}$ 30.000-50.000, $\mathrm{N}$-isopropylacrylamide 97\% (NIPAM), $\mathrm{N}$-isopropylmethacrylate (NIPMAM), 1-vinyl-2-pyrrolidinone (VP), acrylic acid (AAc), potassium persulfate, 2-aminoethyl methacrylate hydrochloride (AEM), triethylamine (TEA), and doxorubicin hydrochloride (DOXO) were supplied by Sigma Aldrich, and acrylamide (AAm) was supplied by BioRad. As solvents, Milli-Q water (18.2 $\mathrm{M} \Omega$, filtered with filter pore size $0.22 \mu \mathrm{M}$ ) from Millipore, acetonitrile (HPLC grade, J. T. Baker), anhydrous tetrahydrofuran, and acetone (Carlo Erba, p.a.) were used. Dynamic light measurements were performed on a Zetasizer Nano ZS90 (Malvern, USA) equipped with a $4.0 \mathrm{~mW} \mathrm{He}-\mathrm{Ne}$ laser operating at $633 \mathrm{~nm}$ and an avalanche photodiode detector. UV-visible absorption spectra were measured using a Varian Cary 5000 UV-vis spectrophotometer equipped with Peltier elements for temperature control. Photoluminescence spectra were recorded on a Cary Eclipse spectrophotometer. FTIR spectra were recorded with a Bruker vertex 70v Fourier transform infrared spectrometer. TEM images were obtained on a JEOL JEM 1011 microscope operated at an accelerating voltage of $100 \mathrm{kV}$. Elemental analysis was carried out via inductively coupled plasma atomic emission spectroscopy on a ThermoFisher CAP 6000 series.

Synthesis of Magnetic Nanobeads. Typically, in an $8 \mathrm{~mL}$ glass vial, $10 \mu \mathrm{L}$ of iron oxide nanoparticles (47 $\mu \mathrm{M}$ nanoparticle 
concentration, $d=6 \mathrm{~nm}$ ) in toluene were dried under a flow of nitrogen, and the IONPs were redissolved in $175 \mu \mathrm{L}$ of THF. Soon after, $25 \mu \mathrm{L}$ of a solution of poly(maleic anhydride-alt-1octadecene) (PC18) in THF (50 mM monomer concentration) was added. The mixture was shaken at $1000 \mathrm{rpm}$ at room temperature for $45 \mathrm{~min}$. After that, $800 \mu \mathrm{L}$ of acetonitrile (ACN) was added to the mixture at a flow rate of $250 \mu \mathrm{L} / \mathrm{min}$. To scale up the synthesis of nanobeads, this procedure was repeated 20 times. (It was not possible to scale up the MNB synthesis just by scaling up the amounts of components in one single reaction pot, as the procedure is very sensitive to air exposure of the polymer used to obtain the beads. The air can affect strongly the hydrolysis of the anhydrides of the polymer and thus the size distribution of the nanobeads. To get a more homogeneous and reproducible MNB batch on which to grow the thermo-responsive shell, the 20 times procedure was therefore preferred.) In order to improve size distribution, after the synthesis of the beads, a magnet $(0.3 \mathrm{~T})$ was placed beneath the vial for $10 \mathrm{~min}$, and the supernatant was recovered, discarding the material trapped on the magnet. The collected supernatant was placed again onto the magnet for $12 \mathrm{~h}$. The supernatant was discarded, and the magnetic material attracted to the magnet was redissolved in fresh acetonitrile. This procedure was repeated three times in order to get pure nanobeads. Finally, the pure nanobeads in acetonitrile were redissolved to a concentration of $529 \mathrm{mg} / \mathrm{L}$ in Fe and an average DLS size of $110 \mathrm{~nm}$ with a polydispersity index (PDI) of 0.094 . The low PDI of the nanobeads obtained by DLS measurements indicates the absence of aggregations. The size, PDI, and Fe concentration of the nanobeads are important parameters to take into account for the functionalization of the bead surfaces with the priming molecules in the following step. The amount of iron was fixed, and a variation on the average bead size $\pm 20 \mathrm{~nm}$ with respect to an average DLS size of $110 \mathrm{~nm}$ was tolerated in order to achieve reproducibility of the procedure.

Functionalization of Nanobeads with AEM Molecules. To $2.4 \mathrm{~mL}$ of a nanobead solution in ACN $(529 \mathrm{mg} / \mathrm{L}$ of Fe and $110 \mathrm{~nm}$ size by DLS) was added $1 \mathrm{~mL}$ of a solution of $10 \mathrm{mg}$ of 2-aminoethyl methacrylate and $16 \mu \mathrm{L}$ of TEA in ACN. Then $12 \mathrm{~mL}$ of anhydrous ACN was added to the mixture, and it was stirred under a nitrogen atmosphere for $24 \mathrm{~h}$ at room temperature. After that, the solution was purified by magnetic attraction (as described above). The precipitate collected on the magnet was fully redissolved in anhydrous $A C N$, and to be sure that the excess AEM was removed, the purification step was repeated twice. Finally, the AEM-MNBs were redissolved in Milli-Q water at a concentration of $412 \mathrm{mg} / \mathrm{L}$ of Fe and had a DLS size of $125 \mathrm{~nm}$ (PDI: 0.066).

Polymerization Procedure on the Magnetic Nanobeads. In a typical polymerization, $8 \mathrm{~mL}$ of degassed water was added to an aqueous solution of AEM-modified nanobead $(0.325 \mathrm{~mL}, 152 \mathrm{mg} / \mathrm{L}$ of $\mathrm{Fe})$ was added $8 \mathrm{~mL}$ of degassed water, and monomers $(0.44 \mathrm{mmol}$ as total amount of monomers having different relative molar ratio composition, see Table 1 ) were collected in a three-necked round-bottom flask under a nitrogen atmosphere. The mixture was heated at $70{ }^{\circ} \mathrm{C}$, and $0.2 \mathrm{~mL}$ of a solution of initiator and sodium persulfate $(10 \mathrm{mg} / \mathrm{mL})$ in degassed water was added. The mixture was stirred for $4 \mathrm{~h}$ at $70{ }^{\circ} \mathrm{C}$ under a nitrogen atmosphere. The polymer MNBs were purified by magnetic separation and were easily redissolved in PBS buffer ( $\mathrm{pH}$ 7.2). This procedure was repeated three times in order to eliminate free monomers, free polymer, initiator, and other impurities. The polymerized nanobeads were analyzed by DLS, TEM, and FTIR, and the LCSTs were determined by turbidimetric analysis.

Dynamic Light Scattering Characterization. The hydrodynamic diameter (measured by DLS) was measured on a solution of MNB-polymers in PBS buffer at a concentration of $50 \mu \mathrm{g} / \mathrm{mL}$ below and above its corresponding LCST (see Figure $3 \mathrm{~A}$ ). The measurements were done on a cell type ZEN0112-low volume disposable sizing cuvette, setting 0.100 as the refractive index and $0.8872 \mathrm{cP}$ as the viscosity. The measurements were performed with $173^{\circ}$ backscatter (NIBS default) as angle of detection, the measurement duration was set as automatic, and the number of measurements was three. As analysis model General Purpose was chosen (normal resolution).
TABLE 1. Initial Molar Composition Chosen for the Growth of the Thermo-responsive Shells at the MNB Surface name

MNB-polymer 1

MNB-polymer 3

MNB-polymer 4

MNB-polymer 5

MNB-polymer 6

MNB-polymer 7

MNB-polymer 8

MNB-polymer 9

MNB-polymer 10

MNB-polymer 11
MNB-polymer 2 monomers

NIPAM

NIPAM/AAm (85:15)

NIPAM/AA (87.5:12.5)

NIPAM/AAm/VP (90:8:2)

NIPAM/AAm/VP (83:8.3:8.3)

NIPAM/AAm/VP (85:5:10)

NIPAM/NIPMAM (1:1)

NIPMAM

NIPMAM/AA (87.5:12.5)

NIPMAM/AAm/VP (85:5:10)

NIPMAM/AAm (85:15) molar composition (mmol)

0.44

$0.374 / 0.066$

$0.38 / 0.055$

$0.396 / 0.035 / 0.0088$

$0.365 / 0.036 / 0.036$

$0.374 / 0.022 / 0.044$

$0.22 / 0.22$

0.44

$0.38 / 0.055$

$0.374 / 0.022 / 0.044$

$0.374 / 0.066$
Trasmission Electron Microscopy Characterization. TEM samples were prepared by dropping a dilute solution of nanobeads on a carbon-coated copper grid and letting the solvent evaporate. The TEM images were taken on the bare MNBs, and the MNBs decorated with different thermo-responsive polymers. The diameters were calculated on an average of 100 MNBs measured.

Turbidimetric Analysis. The turbidimetry was determined by measuring the transmittance of UV-visible light at $400 \mathrm{~nm}$ as a function of temperature. The solution of the thermo-responsive polymer MNBs was transparent and colloidally stable at temperatures below the LCST, and the transmittance was high. Upon increasing the temperature above the phase trasmission temperature, the solution started to get turbid and the transmittance decreased, ${ }^{39}$ due to the corresponding aggregation of polymer MNBs.

Loading of DOXO into MNB-Polymers 8, 9, and 10. $300 \mu \mathrm{L}$ of a DOXO solution $(1 \mathrm{mg} / \mathrm{mL})$ in PBS was added to a $4 \mathrm{~mL}$ solution of MNBpolymer $([\mathrm{Fe}]=50 \mathrm{mg} / \mathrm{L})$ in $\mathrm{PBS}(50 \mathrm{mM}$ and $\mathrm{pH} 7.2)$. The mixture was shaken overnight at room temperature. To remove the unloaded DOXO, the MNBs were centrifuged at $4200 \mathrm{rpm}$ for $5 \mathrm{~min}$, and additionally the vial was placed close to a permanent magnet $(0.3 \mathrm{~T})$ in order to quantitatively separate the MNBs from the solution. The supernatant was collected, and fresh PBS $(4.3 \mathrm{~mL})$ was added to the MNBs. This step was repeated three times in order to remove completely the free DOXO, as confirmed by the absorption spectra (see Figure $7 S$ of the Supporting Information). To validate the amount of DOXO loaded into MNB-polymers 8,9 , and 10, the absorption spectra of the collected supernatants (recovered after having removed the DOXO-loaded MNB-polymer formulations) were recorded. The absorption values at $476 \mathrm{~nm}$ were summed to obtain the free amount of DOXO left in solution. By using the calibration curve of $\mathrm{Abs}(476 \mathrm{~nm})$ vs free DOXO (Figure $6 \mathrm{~S}$ in the $\mathrm{SI}$ ), the concentration of DOXO left in the supernatants was calculated and the concentration of DOXO loaded into MNB-polymers (as well as the loading efficiency) was obtained as the difference between the initial DOXO concentration and that of the supernatant after having removed the DOXO-loaded MNB-polymers. It is worth mentioning that the direct measurement of DOXO loaded within the MNBs is not possible due to the absorption of the MNBs-polymers at the wavelength measured (497 nm).

Release of DOXO in Static Conditions from MNB-Polymers 8, 9, and 10. A solution of DOXO-loaded MNB-polymers ([Fe] $=50 \mathrm{mg} / \mathrm{L}$ ) was incubated at $47^{\circ} \mathrm{C}$ in PBS at $\mathrm{pH}$ 7.2. After defined time lapses the sample was precipitated by centrifugation at $4200 \mathrm{rpm}$ for $5 \mathrm{~min}$, and MNB were attracted to a permanent magnet $(0.3 \mathrm{~T})$. Then the supernatant was collected, and the absorption spectrum was recorded; after that the supernatant was put back and the sample was kept at $47^{\circ} \mathrm{C}$. This step was repeated until enough data points were obtained and the absorption spectra of the supernatant solutions were recorded. To obtain the cumulative release, the absorption values at $476 \mathrm{~nm}$ were summed (data point $1=$ absorption supernatant 1 , data point $2=$ absorption supernatant $1+2$, etc.).

Release of DOXO in the Microfluidic Channel on a Chip. In a typical experiment, $200 \mu \mathrm{L}$ of a MNB-polymer ([Fe] $=50 \mathrm{mg} / \mathrm{L})$ was 
injected in the microfluidic channel via a syringe pump at different flow rates, from 0.8 to $1.2 \mu \mathrm{L} / \mathrm{min}$. The total observation time was $2.5 \mathrm{~h}$ for each flow rate. Videos were taken with a FITC filter in time lapse mode (time step of $5 \mathrm{~min}$, exposure time of $10 \mathrm{~s}$, camera acquisition time of $500 \mathrm{~ms}$ ) in order to avoid photobleaching, possibly due to continuous exposure to excitation light. The exponential decay of the fluorescence intensity from trapped aggregates of MNB has been extracted from videos via ImageJ software. We selected a small area containing a bright spot, and we measured there the maximum and minimum intensity $\left(I_{\max }, I_{\min }\right)$ as a function of time, the latter one being related to the background. In a simple model, $I_{\max }$ and $I_{\min }$ can be written as

$$
\begin{gathered}
I_{\max }=I_{L} h(t) f_{\mathrm{B}}(t) g_{\mathrm{R}}(t) \\
I_{\min }=I_{\mathrm{L}} h(t) f_{\mathrm{B}}(t)
\end{gathered}
$$

where $I_{L}$ is a prefactor taking into account the lamp intensity and the transmission of the optical setup, $h(t) \propto \mathrm{e}^{\left.-\left(t / \tau_{H}\right)\right)}$ is an exponential decay factor due to the non-negligible evaporation, at $45^{\circ} \mathrm{C}$, of the water droplet used for the immersion lens $(60 \times$ water-dipping objective, Eclipse FN1, Nikon, Japan), and $f_{\mathrm{B}}(t)$ describes the evolution of the background, including the possible photobleaching of the solution. Finally $g_{R}(t)$ is proportional to $\mathrm{e}^{-(t / \tau)}$ and describes the fluorescence decay due to the release of the doxorubicin, which can be fitted with an exponential decay having a characteristic decay time $\tau$. By normalizing $I_{\max }$ to $I_{\min }$ we then get $g_{\mathrm{R}}(t)$ and thus $\tau$.

Chip Fabrication. The magnetic array is made of zigzagshaped permalloy $\left(\mathrm{Ni}_{80} \mathrm{Fe}_{20}\right)$ microstripes, $30 \mathrm{~nm}$ thick, patterned on a $\mathrm{Si} / \mathrm{SiO}_{2}(1 \mu \mathrm{m})$ substrate by optical lithography and lift-off. The smallest width of the stripes is $2.5 \mu \mathrm{m}$ and the minimum length of a segment is $12 \mu \mathrm{m}$. The chip surface is then covered with $50 \mathrm{~nm}$ of $\mathrm{SiO}_{2}$ protective layer. A PDMS microfluidic serpentine channel ( $270 \mu \mathrm{m}$ wide and $65 \mu \mathrm{m}$ high) has been fabricated by standard soft lithography techniques and bonded to the chip with magnetic arrays by $\mathrm{O}_{2}$ plasma treatment. The chip is then inserted on a thermostatically controlled heater at the center of a quadrupolar electromagnet controlled by a computer.

Conflict of Interest: The authors declare no competing financial interest.

Supporting Information Available: Additional experimental details including TEM images of nanobeads, size distribution graphs, cloud point measurements, FTIR spectra, dynamic light scattering, calibration curve and absorption spectra of the DOXO release, FITC image, and fluorescence intensity curves at different flow rates. This material is available free of charge via the Internet at http://pubs.acs.org.

Acknowledgment. R.B, A.T., and D.P. thank M. Cantoni and E. Albisetti for fruitful discussions, and M. Leone for his skillful technical support. This work was supported by the European project Magnifyco (Contract NMP4-SL-2009-228622), by the regional project APQ Ricerca Scientifica-Reti di Laboratori Pubblici di Ricerca"—NaBiDiT (p. code 72), by the Italian FIRB project (Nanostructured Oxides, contract no. RBAP115AYN), and by Politecnico di Milano via the project " 5 per mille junior" Magnetically Controlled Single Molecule Delivery (MCSMD). M.P.L. thanks the Junta de Andalucía for a postdoctoral fellowship.

\section{REFERENCES AND NOTES}

1. Petros, R. A.; Desimone, J. M. Strategies in the Design of Nanoparticles for Therapeutic Applications. Nat. Rev. Drug Discovery 2010, 9, 615-627.

2. Gao, X.; Cui, Y.; Levenson, R. M.; Chung, L. W. K.; Nie, S. In Vivo Cancer Targeting and Imaging with Semiconductor Quantum Dots. Nat. Biotechnol. 2004, 22, 969-976.

3. Maeda, H.; Wu, J.; Sawa, T.; Matsumura, Y.; Hori, K. Tumor Vascular Permeability and the EPR Effect in Macromolecular Therapeutics: A Review. J. Controlled Release 2000, $65,271-284$.
4. Kirsebom, H.; Galaev, I. Y.; Mattiasson, B. Stimuli-Responsive Polymers in the 21 st Century: Elaborated Architecture to Achieve High Sensitivity, Fast Response, and Robust Behavior. J. Polym. Sci., Part B: Polym. Phys. 2011, 49, 173178

5. Liu, R.; Fraylich, M.; Saunders, B. R. Thermoresponsive Copolymers: From Fundamental Studies to Applications. Colloid Polym. Sci. 2009, 287, 627-643.

6. Chung, J. E.; Yokoyama, M.; Yamato, M.; Aoyagi, T.; Sakurai, Y.; Okano, T. Thermo-Responsive Drug Delivery from Polymeric Micelles Constructed Using Block Copolymers of Poly(N-Isopropylacrylamide) and Poly(butylmethacrylate) J. Controlled Release 1999, 62, 115-127.

7. Schmaljohann, D. Thermo- and pH-Responsive Polymers in Drug Delivery. Adv. Drug Delivery Rev. 2006, 58, 16551670.

8. Brazel, C. S. Magnetothermally-Responsive Nanomaterials: Combining Magnetic Nanostructures and Thermally-Sensitive Polymers for Triggered Drug Release. Pharm. Res. 2009, 26, 644-656.

9. Figuerola, A.; Di Corato, R.; Manna, L.; Pellegrino, T. From Iron Oxide Nanoparticles towards Advanced Iron-Based Inorganic Materials Designed for Biomedical Applications. Pharmacol. Res. 2010, 62, 126-143.

10. Gazeau, F.; Lévy, M.; Wilhelm, C. Optimizing Magnetic Nanoparticle Design for Nanothermotherapy. Nanomedicine 2008, 3, 831-844.

11. Lee, N.; Hyeon, T. Designed Synthesis of Uniformly Sized Iron Oxide Nanoparticles for Efficient Magnetic Resonance Imaging Contrast Agents. Chem. Soc. Rev. 2012, 41, 25752589.

12. Laurent, S.; Dutz, S.; Häfeli, U. O.; Mahmoudi, M. Magnetic Fluid Hyperthermia: Focus on Superparamagnetic Iron Oxide Nanoparticles. Adv. Colloid Interface Sci. 2011, 166, 8-23.

13. Arruebo, M.; Fernández-Pacheco, R.; Ibarra, M. R.; Santamaría, J. Magnetic Nanoparticles for Drug Delivery. Nano Today 2007, 2, 22-32.

14. Neuberger, T.; Schöpf, B.; Hofmann, H.; Hofmann, M.; Von Rechenberg, B. Superparamagnetic Nanoparticles for Biomedical Applications: Possibilities and Limitations of a New Drug Delivery System. J. Magn. Magn. Mater. 2005, 293, 483-496.

15. Bigall, N. C.; Curcio, A.; Leal, M. P.; Falqui, A.; Palumberi, D.; Di Corato, R.; Albanesi, E.; Cingolani, R.; Pellegrino, T. Magnetic Nanocarriers with Tunable $\mathrm{pH}$ Dependence for Controlled Loading and Release of Cationic and Anionic Payloads. Adv. Mater. 2011, 23, 5645-5650.

16. Riedinger, A.; Pernia Leal, M.; Deka, S. R.; George, C.; Franchini, I. R.; Falqui, A.; Cingolani, R.; Pellegrino, T. "Nanohybrids" Based on pH-Responsive Hydrogels and Inorganic Nanoparticles for Drug Delivery and Sensor Applications. Nano Lett. 2011, 11, 3136-3141.

17. Liu, T. Y.; Hu, S. H.; Liu, D. M.; Chen, S. Y.; Chen, I. W. Biomedical Nanoparticle Carriers with Combined Thermal and Magnetic Responses. Nano Today 2009, 4, 52-65.

18. Pradhan, P.; Giri, J.; Rieken, F.; Koch, C.; Mykhaylyk, O.; Döblinger, M.; Banerjee, R.; Bahadur, D.; Plank, C. Targeted Temperature Sensitive Magnetic Liposomes for ThermoChemotherapy. J. Controlled Release 2010, 142, 108-121.

19. Zhang, X. Z.; Wu, D. Q.; Chu, C. C. Synthesis, Characterization and Controlled Drug Release of Thermosensitive IPNPNIPAAm Hydrogels. Biomaterials 2004, 25, 3793-3805.

20. Liu, T. Y.; Liu, K. H.; Liu, D. M.; Chen, S. Y.; Chen, I. W. Temperature-Sensitive Nanocapsules for Controlled Drug Release Caused by Magnetically Triggered Structural Disruption. Adv. Funct. Mater. 2009, 19, 616-623.

21. Deka, S. R.; Quarta, A.; Di Corato, R.; Riedinger, A.; Cingolani, R.; Pellegrino, T. Magnetic Nanobeads Decorated by Thermo-Responsive PNIPAM Shell as Medical Platforms for the Efficient Delivery of Doxorubicin to Tumour Cells. Nanoscale 2011, 3, 619-629.

22. Chen, S.; Li, Y.; Guo, C.; Wang, J.; Ma, J.; Liangs, X.; Yang, L. R.; Liu, H. Z. Temperature-Responsive Magnetite/PEOPPO-PEO Block Copolymer Nanoparticles for Controlled 
Drug Targeting Delivery. Lanqmuir 2007, 23, 1266912676.

23. Medeiros, S. F.; Santos, A. M.; Fessi, H.; Elaissari, A. StimuliResponsive Magnetic Particles for Biomedical Applications. Int. J. Pharm. 2011, 403, 139-161.

24. Tiera, M. J.; dos Santos, G. R.; Tiera, V. A.; Vieira, N. A. B.; Frolini, E.; da Silva, R. C.; Loh, W. Aqueous Solution Behavior of Thermosensitive (N-Isopropylacrylamide-Acrylic AcidEthyl Methacrylate) Terpolymers. Colloid Polym. Sci. 2005, 283, 662-670.

25. Salehi, R.; Arsalani, N.; Davaran, S.; Entezami, A. A. Synthesis and Characterization of Thermosensitive and $\mathrm{pH}$-Sensitive Poly (N-Isopropylacrylamide-Acrylamide-Vinylpyrrolidone) for Use in Controlled Release of Naltrexone. J. Biom. Mater. Res. A 2009, 89, 919-928.

26. Yang, L.; Guo, C.; Jia, L.; Xie, K.; Shou, Q.; Liu, H. Fabrication of Biocompatible Temperature- and $\mathrm{pH}$-Responsive Magnetic Nanoparticles and their Reversible Agglomeration in Aqueous Milieu. Ind. Eng. Chem. Res. 2010, 49, 8518-8525.

27. Lai, J. J.; Hoffman, J. M.; Ebara, M.; Hoffman, A. S.; Estournès, C.; Wattiaux, A.; Stayton, P. S. Dual Magnetio/TemperatureResponsive Nanoparticles for Microfluidic Separations and Assays. Langmuir 2007, 23, 7385-7391.

28. Donolato, M.; Torti, A.; Kostesha, N.; Deryabina, M.; Sogne, E.; Vavassori, P.; Hansen, M. F.; Bertacco, R. Magnetic Domain Wall Conduits for Single Cell Applications. $\underline{L a b}$ Chip 2011, 11, 2976-2983.

29. Donolato, M.; Gobbi, M.; Vavassori, P.; Leone, M.; Cantoni, M.; Metlushko, V.; Ilic, B.; Zhang, M.; Wang, S. X.; Bertacco, R. Nanosized Corners for Trapping and Detecting Magnetic Nanoparticles. Nanotechnology 2009, 20, 385501(1-6).

30. Di Corato, R.; Bigall, N. C.; Ragusa, A.; Dorfs, D.; Genovese, A.; Marotta, R.; Manna, L.; Pellegrino, T. Multifunctional Nanobeads Based on Quantum Dots and Magnetic Nanoparticles: Synthesis and Cancer Cell Targeting and Sorting. ACS Nano 2011, 5, 1109-1121.

31. Di Corato, R.; Piacenza, P.; Musarò, M.; Buonsanti, R.; Cozzoli, P. D.; Zambianchi, M.; Barbarella, G.; Cingolani, R.; Manna, L.; Pellegrino, T. Magnetic-Fluorescent Colloidal Nanobeads: Preparation and Exploitation in Cell Separation Experiments. Macromol. Biosci. 2009, 9, 952-958.

32. Eeckman, F.; Moës, A. J.; Amighi, K. Synthesis and Characterization of Thermosensitive Copolymers for Oral Controlled Drug Delivery. Eur. Polym. J. 2004, 40, 873-881.

33. Wang, Q.; Zhao, Y.; Xu, H.; Yang, X.; Yang, Y. Thermosensitive Phase Transition Kinetics of Poly(N-Isopropylacryl Amide-co-Acrylamide) Microgel Aqueous Dispersions. J. Appl. Polym. Sci. 2009, 113, 321-326.

34. Chen, H.; Zhu, H.; Hu, J; Zhao, Y.; Wang, Q.; Wan, J.; Yang, Y.; $\mathrm{Xu}, \mathrm{H}$.; Yang, X. Highly Compressed Assembly of Deformable Nanogels into Nanoscale Suprastructures and their Application in Nanomedicine. ACS Nano 2011, 5, 26712680.

35. Kurisawa, M.; Yokoyama, M.; Okano, T. Gene Expression Control by Temperature with Thermo-Responsive Polymeric Gene Carriers. J. Controlled Release 2000, 69, $127-$ 137.

36. Djokpé, E.; Vogt, W. N-Isopropylacrylamide and N-Isopropylmethacrylamide: Cloud Points of Mixtures and Copolymers. Macromol. Chem. Phys. 2001, 202, 750-757.

37. Wan, X.; Liu, T.; Liu, S. Synthesis of Amphiphilic TadpoleShaped Linear-Cyclic Diblock Copolymers via Ring-Opening Polymerization Directly Initiating from Cyclic Precursors and their Application as Drug Nanocarriers. Biomacromolecules 2011, 12, 1146-1154.

38. Cregg, P. J.; Murphy, K.; Mardinoglu, A. Inclusion of Interactions in Mathematical Modelling of Implant Assisted Magnetic Drug Targeting. Appl. Math. Model. 2012, 36, $1-34$.

39. Woodward, N. C.; Chowdhry, B.Z.; Snowden, M. J.; Leharne, S. A.; Griffiths, P. C.; Winnington, A. L. Calorimetric Investigation of the Influence of Cross-linker Concentration on the Volume Phase Pransition of Poly(N-isopropylacrylamide) Colloidal Microgels. Langmuir 2003, 19, 3202-3211. 